\title{
The Future of Social Network Analysis of Health Care System Data: Promise and Concerns
}

\author{
Elizabeth M. Oliva, PhD \\ VA Palo Alto Health Care System, Center for Innovation to Implementation (Ci2i), Menlo Park, CA, USA.
}

J Gen Intern Med 31(2):142-3

DOI: $10.1007 / \mathrm{s} 11606-015-3526-9$

(c) Society of General Internal Medicine 2015

$\mathrm{T}$ he study by Ong et al. ${ }^{1}$ reported in this issue of JGIM represents a major step forward toward improving our understanding of benzodiazepine prescribing and potential targets for improving benzodiazepine prescribing practices. The authors employed social network analysis to model select provider networks and relationships with benzodiazepine prescribing. Their study sought to answer the question, "Does care coordination diminish the chance of multiple-provider prescribing of benzodiazepines?" While this is a laudable study question, it is unclear whether the method employed adequately answers the question. The use of these types of approaches is still in a nascent stage. While these innovative approaches have considerable potential to leverage electronic health record data to improve our understanding of provider and patient networks of care, more research is needed to understand whether these approaches actually measure care coordination.

A previous study ${ }^{2}$ drawing from the same overall data used by Ong et al. ${ }^{1}$ found a great deal of variability in provider patient-sharing networks. The authors commented that "Cohesion among providers is not the norm. Instead, the often ad hoc constellations of providers caring for patients are not persistent teams" (p. 1504). These findings suggest that shared network analysis may not be the best measure of care coordination. Interestingly, in the current study, Ong et al. did include a number of provider-centric measures that may tap into aspects of care coordination (e.g., number of shared patients, percentage of shared patients, Jaccard similarity). Jaccard similarity is a number between 0 and 1 that reflects the degree of similarity in the professional networks of provider pairs (e.g., higher Jaccard similarity scores suggest stronger ties between provider pairs). While these measures were significant, they all had relatively small effect sizes, and the Jaccard similarity measure in particular had questionable clinical significance (e.g., Jaccard similarity mean was 0.38 among provider pairs who did not prescribe overlapping benzodiazepines and 0.35 among those who did). Until research is conducted to validate approaches such as shared network analysis for

Published online October 13, 2015 measuring care coordination, it may be prudent to describe these as measures of patient-sharing networks or "care density" 3,4

While this may seem like a matter of semantics, clarity in constructs is extremely important given the increased interest in measurement-based care and the difficulties with measuring care coordination. ${ }^{5}$ One can imagine a health care system attempting to capture care coordination and cohesion using measures of patient-sharing networks and/or care density, which may be fine given the limited options currently available; however, the limitations of such approaches need to be better understood in order to specify their limitations and to aid in interpreting their findings. Future studies should also investigate the impact of continuous measures of care density (versus dichotomization in the current study) to investigate the independent effect of unit increases in care density on outcomes. These types of studies could help inform policy and staffing decisions when creating care teams.

The rate at which patients received benzodiazepine prescriptions in the Ong et al. study ${ }^{1}$ ( $5.1 \%$ of total patients) is similar to that in a recent study by Olfson et al., ${ }^{6}$ which estimated that $5.2 \%$ of adults in the United States in 2008 received prescriptions for benzodiazepines. Interestingly, Olfson et al. found that most benzodiazepine prescribing was done by non-psychiatrists. Although Ong et al. found that the presence of a psychiatrist on a patient's care team was associated with a greater likelihood of receiving of an overlapping benzodiazepine prescription (defined as having overlapping coverage of 14 or more days), it was not clear whether the psychiatrist was involved in prescribing. However, Ong et al. did find that having benzodiazepines prescribed by a primary care provider was associated with overlapping benzodiazepine prescription. In light of the finding by Olfson et al. suggesting that psychiatrists might prescribe benzodiazepines more appropriately than non-psychiatrists, it would be helpful to understand the impact of psychiatrist involvement in care teams on prescribing behaviors, as well as the source of the overlapping prescription (e.g., whether it was prescribed first by a psychiatrist and then by a primary care provider [PCP] or vice versa). More research is needed to better understand these relationships and to develop methods to improve medication safety.

Notably, pharmacists could be an important target given that, at least in the United States, they are responsible for the vast majority of medication dispensing. For instance, Ong 
et al. ${ }^{1}$ found that among patients with benzodiazepine prescriptions (5.1\% of total patients in the study sample), multiple prescriptions were found among $21 \%(1.1 \%$ of total patients) and overlapping prescriptions among $3.8 \%(0.2 \%$ of total patients). Given the relatively low base rates of these prescribing practices, other approaches are clearly needed to improve medication safety. Although benzodiazepine prescribing in itself is concerning, recent studies have also highlighted the risks associated with co-prescribed benzodiazepines and opioids. In an analysis of the period between 2004 and 2011, Jones et al. ${ }^{7}$ linked the combined use of opioid analgesics and benzodiazepines to a more than threefold increase in the rate of emergency department visits, and a similar increase in the rate of overdose deaths. Among patients treated in the Veterans Health Administration between 2004 and 2009, Park et al. ${ }^{8}$ found that $27 \%$ of those who received opioid analgesics also received benzodiazepines, and that $49 \%$ of deaths from drug overdose occurred during the period of co-prescription.

In response to the increasing concerns about medication safety, health care systems have developed system-level approaches for improving safety that include provider-level interventions (e.g., Veterans Health Administration's Opioid Safety Initiative and Psychotropic Drug Safety Initiative). Social network analyses represent one promising method for examining how system-level changes are adopted by providers and provider networks (e.g., diffusion of best practices) and impact quality of care. ${ }^{5}$ Moreover, these approaches may be helpful in targeting key providers and provider networks, given that $31 \%$ of provider pairs in this study accounted for $100 \%$ of overlapping benzodiazepine prescribing. One promising approach published recently involves the use of Prescription Drug Monitoring Programs (PDMPs) to develop algorithms for identifying providers with unusual prescribing practices. ${ }^{9}$ The authors of the study suggest ways to use the algorithms to improve prescribing practices via motivation, enforcement and remediation (e.g., automated alerts to providers, involvement of licensing boards, tailored education). A multifaceted approach will likely be needed to fully address provider prescribing practices.

One caveat should be kept in mind when interpreting the results of the study by Ong et al. ${ }^{1}$ While the authors did not find the expected association between comorbidity and overlapping benzodiazepine prescriptions, they examined only medical comorbidity and not mental health comorbidity, and only patients between the ages 18 and 64 were included. Given that the study by Olfson et al. ${ }^{6}$ suggests that the highest rates of benzodiazepine use were among 65 - to 80 -year-olds, Ong et al.'s study excludes an important sub-population that may be at greater risk for overlapping benzodiazepine prescriptions, and may underestimate the potential effects of medical comorbidities. Moreover, in light of the indications for benzodiazepines, future studies should include measures of mental health comorbidities.

Nonetheless, the study by Ong et al. increases our understanding of benzodiazepine prescribing, and their approach sheds light on provider as well as patient behaviors. I hope to see more research in this area in the near future.

Acknowledgments: The views expressed here are the author's and do not necessarily represent those of the Department of Veterans Affairs. The author would like to thank Jodie Trafton and Eleanor Lewis for their feedback on various aspects of this editorial.

\section{Compliance with Ethical Standards}

Conflict of Interest: The author declares that she does not have a conflict of interest.

Corresponding Author: Elizabeth M. Oliva, PhD; VA Palo Alto Health Care SystemCenter for Innovation to Implementation (Ci2i), 795 Willow Road (152 MPD), Menlo Park, CA 94025, USA (e-mail: elizabeth.oliva@va.gov).

\section{REFERENCES}

1. Ong MS, Olson KL, Cami A, Liu C, Tian F, Selvam N, Mandl KD. Provider patient-sharing networks and multiple-provider prescribing of benzodiazepines. J Gen Intern Med. doi: 10.1007/s11606-015-3470-8.

2. Mandl KD, Olson KL, Mines D, Liu C, Tian F. Provider collaboration: cohesion, constellations, and shared patients. J Gen Intern Med. 2014;29: 1499-505.

3. Landon BE, Keating NL, Barnett ML, Onnela JP, Paul S, O'Malley AJ, Keegan T, Christakis NA. Variation in patient-sharing networks of physicians across the United States. JAMA. 2012;308:265-73.

4. Pollack CE, Weissman GE, Lemke KW, Hussey PS, Weiner JP. Patient sharing among providers and costs of care: a network analytic approach to care coordination using claims data. J Gen Intern Med. 2013;28:459-65.

5. Bynum JP, Ross JS. A measure of care coordination? J Gen Intern Med. 2013;28:336-8.

6. Olfson M, King M, Schoenbaum M. Benzodiazepine use in the United States. JAMA Psychiatry. 2015;72:136-42.

7. Jones CM, McAninch JK. Emergency department visits and overdose deaths from combined use of opioids and benzodiazepines. Am J Prev Med. doi: 10.1016/j.amepre.2015.03.040.

8. Park TW, Saitz R, Ganoczy D, Ilgen MA, Bohnert ASB. Benzodiazepine prescribing patterns and deaths from drug overdose among US veterans receiving opioid analgesics: case-cohort study. BJM. 2015;350:h2698.

9. Ringwalt C, Schiro S, Shanahan M, Proescholdbell S, Meder H, Austin A, Sachdeva N. The use of a prescription drug monitoring program to develop algorithms to identify providers with unusual prescribing practices for controlled substances. J Prim Prev. doi: 10.1007/s10935-015-0397-0. 